\title{
The Trend and Age-Period-Cohort Analysis on the Burden of Cardiovascular Disease attributable to Tobacco Exposure in China, Japan, USA and World
}

\author{
Wenying Wu \\ The First Hospital of China Medical University \\ Bo Zhu \\ Liaoning Cancer Institute and Hospital \\ Shuang Xu \\ library of China medical university \\ Yong Liu \\ Saarland university \\ Yifei Bi \\ leiden university \\ xiaomei wu ( $\nabla$ wxm_555555@sina.com )
}

the first hospital of china medical university https://orcid.org/0000-0002-8406-0005

\section{Research article}

Keywords: Tobacco exposure, Cardiovascular Disease, Age-Period-Cohort Analysis

Posted Date: October 11th, 2019

DOI: https://doi.org/10.21203/rs.2.15226/v2

License: (c) (1) This work is licensed under a Creative Commons Attribution 4.0 International License. Read Full License 


\section{Abstract}

Background Tobacco exposure is the major contributor of CVD mortality, but none of the published studies on CVD mortality attributable to tobacco exposure analyzed the possible reasons underlying the long-term trends and the differences between age-groups in China.Methods The mortality data in China, Japan, USA and World were obtained from GBD 2017. The joinpoint regression analysis was used to assess the magnitude and direction of trends over time for CVD morality, and the age-period-cohort method was used to analyze the temporal trends of CVD mortality by age, period, and cohort. The smoking prevalence was acquired from WHO. Results There was a significant downward trend in ASMR of CVD attributable to smoking in four regions, but China has the smallest decline and the ASMR of CVD attributable to smoking in China rose to the first place in 2017 (41.41 per 100,000). All the net drifts per year in four regions were negative, and the local drift values were below zero in all age groups. The longitudinal age curves of the CVD mortality attributable to smoking increased in four regions and China had the largest increase. The period/cohort RRs indicated a decline, and China has the smallest decline. All the cohort/period RRs and net drifts per year in female had more quickly declining trends than that in male. All the results of secondhand smoke were similar to that of smoking. For Stroke and IHD, their results were similar to those of CVD.Conclusions Although CVD mortality attributable to tobacco exposure had declined in four regions, CVD mortality attributable to tobacco exposure in China was at a high level, and the high CVD mortality attributable to secondhand smoke caused by high smoking prevalence. China should strengthen tobacco control measures to reduce the burden of CVD.

\section{Background}

Accompanying economic development and urbanization, the patterns of disease has shifted from infectious diseases to noncommunicable diseases. As one of noncommunicable diseases, cardiovascular disease (CVD) is the leading cause of premature morbidity and mortality in the world. In 2017, the estimated 17.8 million people died of CVD, which was the cause of $30 \%$ of deaths all over the world[1]. In China, CVD mortality had increased from 187.58 per 100,000 in 1990 to 309.95 per 100,000 in 2017, and the burden of CVD was also very high, the estimated number of CVD death had almost doubled, from 2.25 million in 1990 to 4.38 million in 2017[2]. In the world, China has the highest burden of CVD, China is facing enormous challenges in tackling the burden of CVD [3].

Tobacco exposure mainly included active smoking and secondhand smoke (SHS). In the world, nearly 7 million people died from tobacco exposure, including approximately 6 million among tobacco users and an estimated 890,000 among nonsmokers exposed to SHS [4]. Longterm smokers lost at least 10 years of life on average. In the world, over 22000 people die from tobacco exposure every day (one person dies every 4 seconds). Smoking is a widely recognized risk factor for premature morbidity and mortality, but adequate monitoring of smoking levels and trends throughout the world has been challenging. Nearly two-thirds of smokers in the world were mainly in ten countries, and China accounted for about $30 \%$. In 2017, active smoking had become the first leading risk factor for DALYs and the second risk factor for death in China [5].

Tobacco exposure is a major modifiable risk factor for CVD. Several epidemiological studies concluded that smoking is a major cause of CVD and causes one of every four deaths from CVD[6]. SHS also can cause CVD, including heart attack and stroke. Nonsmokers who breathe SHS at home or at work increase their risk of developing heart disease by $25-30 \%$. SHS increases the risk for stroke by $20-30 \%[7]$. The World Health Organization (WHO) considered that Tobacco exposure was the totally avoidable risk factor of CVD[8]. The probability of tobacco exposure varied by different age and gender, and the different regions had different tobacco control strategies, so the disease burden attributable to tobacco exposure varied among age, sex and regions[9]. Currently, none of the published studies on CVD mortality attributable to tobacco exposure had analyzed the possible reasons underlying the long-term trends and the differences between age-groups. Therefore, the objective of our study was to compare CVD mortality attributable to tobacco exposure in China, Japan, United States (USA) and the world during the period between 1990 and 2017, and analyzed the changes in long-term trends and the independent effects of chronological age, time period, and birth cohort, and provided evidences for tobacco control strategies.

\section{Methods}

\section{Data Source}

Data are available at the GBD Data Tool repository and can be accessed at http://ghdx.healthdata.org/gbd-results-tool. To analyze the status on CVD mortality attributable to tobacco exposure in China, we extract the relevant data on CVD mortality, and compared to Japan, USA and the world. In 2017, ischemic heart disease (IHD) and stroke were top two in mortality rate of all the causes of death in China[2], so we not only analyzed the changes of CVD attributable to tobacco exposure by the temporal trend and Age-Period-Cohort methods, but also analyzed the changes of IHD and stroke, in order to reflect the impact of tobacco exposure on CVD systematically and comprehensively. We used four outcome measures in APC model for evaluation: (1) the fitted longitudinal age-specific rates in the reference cohort adjusted for period deviations; (2) the cohort/period rate ratios (RR) adjusted for age and nonlinear cohort/period effects in a cohort/period versus the reference one; (3) the local drifts which show the annual percentage changes for each age group; (4) the net drift which show the overall annual 
percentage change. CVD, IHD and stroke were diagnosed and defined based on the WHO clinical criteria and ICD 9. The smoking prevalence in four regions was collected from $\mathrm{WHO}[10]$. The dataset provided the summarized smoking prevalence for each country in four year points (2000, 2005, 2010 and 2015). Please see the detailed data in Additional file 1-Table S1.

\section{Statistical Analysis}

In order to assess the magnitude and direction of trends over time for morality rate, we used JoinPoint software (Version 4.7.0.0) to calculate the average annual percentage change (AAPC) and the corresponding $95 \%$ Cls by joinpoint regression analysis. We used three joinpoints to detect four different trends uniformly, to investigate the trend of mortality rate of CVD attributable to tobacco exposure in different periods.

In order to assess death risks experienced by the population in a particular year and the accumulation of health risks since birth, we used the age-period-cohort (APC) model to analyze the temporal trends by age, period, and cohort and after adjustment for age, period, and cohort on smoke-attributable CVD mortality. The APC model could provide a useful parametric framework that complements standard nonparametric descriptive methods. In our APC model, we needed to convert the collected data to successive 5-year age groups and consecutive 5-year periods. Because the GBD dataset did not provide successive 5-year age groups under 29 or over 80 years in smoking, the mortality rates of CVD attributable to smoking were recoded into successive 5-year age groups (30-34 years to 75-79 years) and consecutive 5-year periods (1990 to 2017). Because the GBD dataset did not provide successive 5-year age groups under 25 or over 80 years in SHS, the mortality rates of CVD attributable to SHS were recoded into successive 5-year age groups (25-29 years to 75-79 years) and consecutive 5-year periods (1990 to 2017). The general linear model was used to analyze the slope of the period/cohort RRs. The statistical analysis was performed by $R$ statistical software ( $R$ version 3.5.1), and $p<0.05$ was considered significant.

\section{Results}

\section{(1) The temporal trend in the age-standardized mortality rate (ASMR) of CVD attributable to Tobacco Exposure from 1990 to 2017}

Smoking: For both sexes, in 1990, the rank in the ASMR of CVD attributable to smoking was USA, world, China and Japan. In 2017, the rank was China, world, USA and Japan. The ASMR of CVD attributable to smoking in four regions significantly decreased by $0.4 \%$ ( $95 \% \mathrm{Cl}$ : $0.0 \%-0.7 \%), 4.0 \%$ (95\% Cl: 3.7\%-4.3\%), 4.9\% (95\% Cl: 4.7\%-5.1\%) and 2.1\% (95\% Cl: 1.8\%-2.4\%) per year, respectively. For male, the rank in 1990 and 2017 were similar to both sexes. There was a significant downward trend in USA, world and Japan, but there was no significant downward trend in China. For female, in 1990, the rank was USA, world, Japan and China. In 2017, the rank was USA, China, world and Japan. There was a significant downward trend in USA, China, world and Japan. Figure 1 and Table 1 showed the temporal trend in ASMR of CVD attributable to smoking in China, Japan, USA and the world from 1990 to 2017.

Secondhand smoke: For both sexes, from 1990 to 2017, there was no change in the rank for the ASMR of CVD attributable to SHS with China on top followed by world, USA and Japan. The ASMR of CVD attributable to SHS in China, Japan, the USA and the world significantly decreased by $1.2 \%$ (95\% Cl: $0.7 \%-1.7 \%)$, 3.8\% (95\% Cl: 3.5\%-4.1\%), 3.9\% (95\%Cl: $3.6 \%-4.2 \%)$ and $1.8 \%(95 \% \mathrm{Cl}$ : $1.5 \%-2.0 \%)$ per year, respectively. For male, the rank in 1990 was world, China, USA and Japan. In 2017, the rank in the ASMR of CVD attributable to SHS was China, world, USA and Japan. There was a significant downward trend in USA, world and Japan, but there was no significant downward trend in China. For female, the rank from 1990 to 2017 was similar to both sexes. There was a significant downward trend in USA, China, world and Japan. Figure 2 and Table 2 showed the temporal trend in ASMR of CVD attributable to SHS in China, Japan, USA and the world from 1990 to 2017.

\section{(2) The APC analysis in the mortality rate of CVD attributable to Tobacco Exposure from 1990 to 2017}

Smoking: For both sexes, in the same birth cohort, the mortality rate of CVD attributable to smoking rapidly increased 7.38 (95\%Cl: $6.34,8.58$ ) per 100,000 in age group $30-34$ to 360.15 (95\% Cl: $338.97,382.67)$ per 100,000 in age group $75-79$ in China. The similar changes can be observed in world, USA and Japan. Figure 3(A) showed the longitudinal age curves of the mortality rate of CVD attributable to smoking. All the cohort and period RRs showed a decreasing trend in four regions. For male and female, the changes in mortality rate were similar to both sexes. All the cohort and period RRs were statistically significant $(P<0.001$ for all) and so were the net drifts. Figure 3(B, C) showed the estimated cohort and period RRs of the mortality rate of CVD attributable to smoking. Figure 4(A) and Table 3 showed the detailed results of net drifts.

Secondhand smoke: All the results were similar to that of smoking. Figure 5(A) showed the longitudinal age curves of the mortality rate of CVD attributable to SHS. Figure 5(B, C) showed the estimated cohort and period RRs of the mortality rate of CVD attributable to SHS. Figure 4(B) and Table 3 showed the detailed results of net drifts. 
(3) The temporal trend in the age-standardized mortality rate (ASMR) of stroke attributable to Tobacco Exposure from 1990 to 2017

Smoking: The rank in stroke was slightly different from CVD from 1990 to 2017. For both sexes, the rank in the ASMR of stroke attributable to smoking was China, world, Japan and USA all the time. There was a significant downward trend in four regions. The temporal trends in male and female were similar to both sexes. Additional file 2-Figure S1 and Additional file 1-Table S2 showed the temporal trend in ASMR of stroke attributable to smoking in China, Japan, USA and the world from 1990 to 2017.

Secondhand smoke: All the results were similar to that of smoking. Additional file 2-Figure S2 and Additional file 1-Table S3 showed the temporal trend in ASMR of stroke attributable to SHS in China, Japan, USA and the world from 1990 to 2017.

\section{(4) The APC analysis in the mortality rate of stroke attributable to Tobacco Exposure from 1990 to 2017}

Smoking: For both sexes, in the same birth cohort, the mortality rate of stroke attributable to smoking rapidly increased in China. The similar changes can be observed in other three regions. Additional file 2-Figure S3(A) showed the longitudinal age curves of the mortality rate of stroke attributable to smoking. All the cohort and period RRs showed a decreasing trend in four regions. For male and female, the changes in mortality rate were similar to both sexes. All the cohort and period RRs were statistically significant $(P<0.001$ for all $)$ and so were the net drifts. Additional file 2-Figure S3(B, C) showed the estimated cohort and period RRs of the mortality rate of stroke attributable to smoking. Additional file 2-Figure S4(A) and Additional file 1-Table S4 showed the detailed results of net drifts.

Secondhand smoke: All the results were similar to that of smoking. Additional file 2-Figure S5(A) showed the longitudinal age curves of the mortality rate of stroke attributable to SHS. Additional file 2-Figure S5(B, C) showed the estimated cohort and period RRs of the mortality rate of stroke attributable to SHS. Additional file 2-Figure S4(B) and Additional file 1-Table S4 showed the detailed results of net drifts.

\section{(5) The temporal trend in the age-standardized mortality rate (ASMR) of IHD attributable to Tobacco Exposure from 1990 to 2017}

Smoking: The rank in IHD was similar to CVD from 1990 to 2017. For both sexes, in1990, the rank in the ASMR of IHD attributable to smoking was USA, world, Japan and China. In 2017, the rank was China, world, USA and Japan. There was a significant downward trend in world, USA and Japan, but there was a significant upward trend in China, especially in 1999-2015. For male, the temporal trend was similar to both sexes. Although the mortality rate in China is rising, it is not significant. For female, there was a significant downward trend in world, USA and Japan. Additional file 2-Figure S6 and Additional file 1-Table S5 showed the temporal trend in ASMR of IHD attributable to smoking in China, Japan, USA and the world from 1990 to 2017.

Secondhand smoke: For both sexes, in 1990, the rank in the ASMR of IHD attributable to second smoke was world, China, USA and Japan. In 2017, the rank was China, world, USA and Japan. There was a significant downward trend in world, USA and Japan, but there was no significant trend change in China. The temporal trends in male and female were similar to both sexes. Additional file 2-Figure S7 and Additional file 1-Table S6 showed the temporal trend in ASMR of IHD attributable to SHS in China, Japan, USA and the world from 1990 to 2017.

\section{(6) The APC analysis in the mortality rate of IHD attributable to Tobacco Exposure from 1990 to 2017}

Smoking: For both sexes, in the same birth cohort, the mortality rate of IHD attributable to smoking rapidly increased $3.39(95 \% \mathrm{Cl}: 2.90,3.35)$ per 100,000 in age group 30-34 to 192.20 (95\% Cl: $179.44,205.86)$ per 100,000 in age group 75-79 in China. The similar changes can be observed in world, USA and Japan. Additional file 2-Figure S8(A) showed the longitudinal age curves of the mortality rate of stroke attributable to smoking. All the cohort and period RRs showed a decreasing trend in four regions. For male and female, the changes in mortality rate were similar to both sexes. The cohort and period RRs were statistically significant in world, USA and Japan $(P<0.001$ for all) and so were the net drifts. Additional file 2-Figure $S 8(B, C)$ showed the estimated cohort and period RRs of the mortality rate of CVD attributable to smoking. Additional file 2-Figure S9(A) and Additional file 1-Table S7 showed the detailed results of net drifts.

Secondhand smoke: All the results were similar to that of smoking. Additional file 2-Figure S10(A) showed the longitudinal age curves of the mortality rate of IHD attributable to SHS. Additional file 2-Figure S10 $(B, C)$ showed the estimated cohort and period RRs of the mortality rate of CVD attributable to SHS. Additional file 2-Figure S9(B) and Additional file 1-Table S7 showed the detailed results of net drifts.

\section{Discussion}

Our results show that for CVD, there was a significant downward trend in ASMR of CVD attributable to smoking from 1990 to 2017 in four regions, but China has the smallest decline. Therefore, the rank in the ASMR of CVD attributable to smoking had changed, China rose to the 
first place in 2017. The ASMR of CVD attributable to smoking in Japan was at the lowest level in four regions. We found that the longitudinal age curves of the mortality rate of CVD attributable to smoking increased in four regions, and China had the largest increasing extent.

Regardless of age and birth cohort effects, the period RRs indicated a decline in the mortality rate of CVD attributable to smoking in recent years. Similarly, regardless of the age and period effects, the cohort RRs also indicated a decline in CVD mortality among younger generations. All the net drifts were significant. China has the smallest decline in the cohort and period RRs, and all the cohort and period RRs in female has more quickly declining trends than that in male. All the results of SHS were similar to that of smoking.

Because stroke and IHD were important components of CVD, we further analyzed the trend of their ASMR attributable to tobacco exposure and the impact of age, cohort and period on mortality rate. For stroke, the rank in the ASMR of stroke attributable to smoking had no change, China remained the first place from 1990 to 2017. The downward trend in ASMR, the change of the cohort and period RRs and the net drifts of stroke were similar to those of CVD. For IHD, we found that the downward trends in ASMR, the change of the cohort and period RRs and the net drifts of IHD were similar to those of CVD in the world, USA and Japan. In China, the significant upward trend in both sexes and male were observed, but all the cohort and period RRs in female has more quickly declining trends.

Tobacco exposure can damage blood vessels very quickly, but the blood vessels can repair themselves naturally when smokers stop smoking, heart attack risk drops dramatically in one year, and risk of stroke can reduce to the level of nonsmoker[11]. Tobacco exposure caused CVD by atherosclerosis and thrombosis. The mechanism of smoking-induced atherosclerosis and thrombosis was that oxidative stress mediated by cigarette smoke and/or endogenous oxygen free radicals, multiple thrombosis-promoting and anti-fibrinolysis effects, and lead to intravascular thrombosis and trigger acute cardiovascular events[12, 13]. Free radicals may originate from: (1) gases or tar in cigarette smoke, (2) circulating or in situ-activated macrophages and neutrophils, (3) endogenous sources of reactive oxygen species such as uncoupled eNOS and the mitochondrial electron transport chain. The free radicals, such as superoxide, reacted with NO, this not only reduced the utilization of NO, but also produced peroxide nitrite, which further enhanced oxidative stress in cells[14, 15]. Eventually, tobacco exposure can lead to plaque and blood clots, restrict blood flow, and lead to heart attack and stroke. Stroke, like heart disease, has a high risk of death, and survivors of stroke may experience disastrous disabling conditions, such as paralysis or loss of vision or speech [16].

One in three deaths is attributed to CVD in the world, and tobacco exposure is the major contributor, causing approximately 3 million cardiovascular deaths every year in the world [17]. In 2019, WHO reported estimates of the smoking prevalence for all countries on the global tobacco epidemic[10]. From the report, we found that compared with other three regions, the smoking prevalence in China had been at highest level, and the decline of smoking prevalence in China was the smallest, the exposure of second-hand smoke would also be at a high level, so the ASMR of stroke, IHD and CVD caused by tobacco exposure would also be higher, and the decline of ASMR would be smaller. These were concluded by our results, and tobacco exposure had caused a great burden on CVD mortality in China. Smoking increased the risk of CVD, and so did SHS, but the hazards of SHS were often ignored. In our study, from 1990 to 2017, the ratio of second-hand smoke to smoking in the ASMR of CVD ranged from 1/3 to 1/4 in China. Compared with other three regions, the ratio of China was highest, SHS exposure caused a serious burden of CVD in China. A study on SHS found that an almost $40 \%$ increase in mortality risk among non-smoking women whose husbands smoked, and the incidence of heart disease increased[18]. A national population-based case-control study found that SHS could increase the risk of death by $10 \%$ for all strokes by adjustment for related variables [19].

In age effect, we found that the CVD (including stroke and IHD) mortality attributable to tobacco exposure increased with age. This might be mainly caused by two reasons: On the one hand, long-term tobacco exposure led to vascular damage; on the other hand, physical resistance declined with age[20]. Due to the possibility of tobacco exposure, sex was one of important demographic risk factor for CVD [21]. In four regions, the smoking prevalencein male was higher than that in female, the difference in smoking rates between men and women in China was the largest, and so was the difference in ASMR attributable to smoking. In our study, we found that the changes of ASMR attributable to smoking for both sexes were similar to those for male, and the changes of ASMR attributable to SHS for both sexes were similar to those for female. In the cohort effect, we found that the later the year of birth, the lower the mortality rates. In the period effect, we also found that the mortality rates gradually decreased overtime. By the development of the economy, social propaganda, and policy constraints, people had a certain level of understanding of the dangers of tobacco exposure, resulting in lower smoking prevalence, and lower exposure to second-hand smoke in public areas, resulting in reduced the mortality rate of CVD attributable to tobacco exposure[22].

Our study is the first study to show the temporal trends of CVD mortality attributable to tobacco exposure, to analyze the effects of age-cohortperiod, and to focus on a comprehensive comparison in China, Japan, USA and the world. The ASMRs, period effects and cohort effects of CVD mortality attributable to tobacco exposure in four regions have been declining in both sexes and all age groups from 1990 to 2017. Comparing with the differences between their results, we found the gap between different regions: the USA and Japan had greatly reduced the CVD mortality attributable to tobacco exposure from 1990 to 2017, which were lower than the world average levels, and should continue to maintain the status. The CVD mortality attributable to tobacco exposure in China was higher than that in the world. Based on the above findings, China should try its best to implement tobacco control strategies to reduce the effect of tobacco exposure on CVD mortality in the next few years, to achieve the goal of reducing the burden of CVD. 


\section{Conclusion}

Tobacco control strategies were of low cost and effective for the prevention of CVD across a range of different environments. Our study contributed to the importance of implementing strong tobacco. Although CVD mortality attributable to tobacco exposure had declined in four regions, CVD mortality attributable to tobacco exposure in China was at a high level, and the high CVD mortality attributable to secondhand smoke caused by high smoking prevalence. China should strengthen tobacco control measures to achieve the goal of reducing the burden of CVD.

\section{List Of Abbreviations}

Cardiovascular Disease: CVD

Secondhand smoke: SHS

World Health Organization: WHO

Ischemic heart disease: IHD

Average annual percentage change: AAPC

The age-period-cohort: APC

Age-standardized mortality rate: ASMR

\section{Declarations}

Ethics approval and consent to participate

GBD 2017 was publicly available for free use, the protocol was approved by the Medical Research Ethics Committee of the First Hospital of China Medical University (2015-149).

\section{Consent for publication}

Not applicable

\section{Availability of data and materials}

All our research data are obtained from GBD 2017, the website was http://ghdx.healthdata.org/ gbd-results-tool.

\section{Competing Interests}

The authors declare that they have no competing interests.

\section{Funding}

This work was supported by General Foundation of scientific research in the Department of Education in Liaoning (L2015592).

\section{Author Contributions}

XMW and $B Z$ designed the whole research, WY W, S X, and $Y$ L conducted the data collection, WY W, B Z and XMW analyzed the data. B Z, YF $B$ and $X M$ W wrote the manuscript. WY W, B Z, S X, Y L, and XM W discussed the relevant results.

\section{Acknowledgements}

Not applicable.

\section{References}

1. Jagannathan R, Patel SA, Ali MK, Narayan KMV: Global Updates on Cardiovascular Disease Mortality Trends and Attribution of Traditional Risk Factors. Current diabetes reports 2019, 19(7):44.

2. Global Health Data Exchange. GBD Results Tool [http://ghdx.healthdata.org/ gbd-results-tool] 
3. Du X, Patel A, Anderson CS, Dong J, Ma C: Epidemiology of Cardiovascular Disease in China and Opportunities for Improvement: JACC International. Journal of the American College of Cardiology 2019, 73(24):3135-3147.

4. GBD 2015 Tobacco Collaborators: Smoking prevalence and attributable disease burden in 195 countries and territories, 1990-2015: a systematic analysis from the Global Burden of Disease Study 2015. Lancet (London, England) 2017, 389(10082):1885-1906.

5. Zhou M, Wang H, Zeng X, Yin P, Zhu J, Chen W, Li X, Wang L, Wang L, Liu Y et al: Mortality, morbidity, and risk factors in China and its provinces, 1990-2017: a systematic analysis for the Global Burden of Disease Study 2017. Lancet (London, England) 2019.

6. National Center for Chronic Disease Prevention and Health Promotion (US) Office on Smoking and Health: The Health Consequences of Smoking-50 Years of Progress: A Report of the Surgeon General. Atlanta GA; 2014.

7. Centers for Disease Control and Prevention (CDC) United States: Vital signs: nonsmokers' exposure to secondhand smoke -- United States, 1999-2008. MMWR Morbidity and mortality weekly report 2010, 59(35):1141-1146.

8. Mannan H, Stevenson C, Peeters A, Walls H, McNeil J: Framingham risk prediction equations for incidence of cardiovascular disease using detailed measures for smoking. Heart international 2010, 5(2):e11.

9. Brown Q, Milam AJ, Bowie JV, lalongo NS, Gaskin DJ, Furr-Holden D: The Moderating Role of Gender in the Relationship Between Tobacco Outlet Exposure and Tobacco Use Among African American Young Adults. Prevention science : the official journal of the Society for Prevention Research 2016, 17(3):338-346.

10. World Health Organization: WHO global report on trends in prevalence of tobacco smoking 2000-2025. 2018.

11. Roelsgaard IK, Esbensen BA, Ostergaard M, Rollefstad S, Semb AG, Christensen R, Thomsen T: Smoking cessation intervention for reducing disease activity in chronic autoimmune inflammatory joint diseases. The Cochrane database of systematic reviews 2019 , 9:CD012958.

12. Ambrose JA, Barua RS: The pathophysiology of cigarette smoking and cardiovascular disease: an update. Journal of the American College of Cardiology 2004, 43(10):1731-1737.

13. Messner B, Bernhard D: Smoking and cardiovascular disease: mechanisms of endothelial dysfunction and early atherogenesis. Arteriosclerosis, thrombosis, and vascular biology 2014, 34(3):509-515.

14. DiGiacomo SI, Jazayeri MA, Barua RS, Ambrose JA: Environmental Tobacco Smoke and Cardiovascular Disease. International journal of environmental research and public health 2018, 16(1).

15. Sandler P, Mastella B, Uchoa D, Jotz GP, Leao HZ, Cavazzola LT: The effects of passive tobacco smoking on the microcirculation of the abdominal wall in rats. Acta cirurgica brasileira 2016, 31(11):714-719.

16. Benowitz NL, Burbank AD: Cardiovascular toxicity of nicotine: Implications for electronic cigarette use. Trends in cardiovascular medicine 2016, 26(6):515-523.

17. Rigotti NA, Clair C: Managing tobacco use: the neglected cardiovascular disease risk factor. European heart journal 2013, 34(42):32593267.

18. Gan Q, Smith KR, Hammond SK, Hu TW: Disease burden of adult lung cancer and ischaemic heart disease from passive tobacco smoking in China. Tobacco control 2007, 16(6):417-422.

19. Hou L, Han W, Jiang J, Liu B, Wu Y, Zou X, Xue F, Chen Y, Zhang B, Pang H et al: Passive smoking and stroke in men and women: a national population-based case-control study in China. Scientific reports 2017, 7:45542.

20. Heitzer T, Meinertz T: [Prevention of coronary heart disease: smoking]. Zeitschrift fur Kardiologie 2005, 94 Suppl 3:Ill/30-42.

21. Chiuve SE, Cook NR, Shay CM, Rexrode KM, Albert CM, Manson JE, Willett WC, Rimm EB: Lifestyle-based prediction model for the prevention of CVD: the Healthy Heart Score. Journal of the American Heart Association 2014, 3(6):e000954.

22. Zhang J, Ou JX, Bai CX: Tobacco smoking in China: prevalence, disease burden, challenges and future strategies. Respirology (Carlton, Vic) $2011,16(8): 1165-1172$.

\section{Tables}

Table 1. The temporal trend in mortality rate of CVD attributable to smoking in China, Japan, USA and the world from 1990 to 2017. 


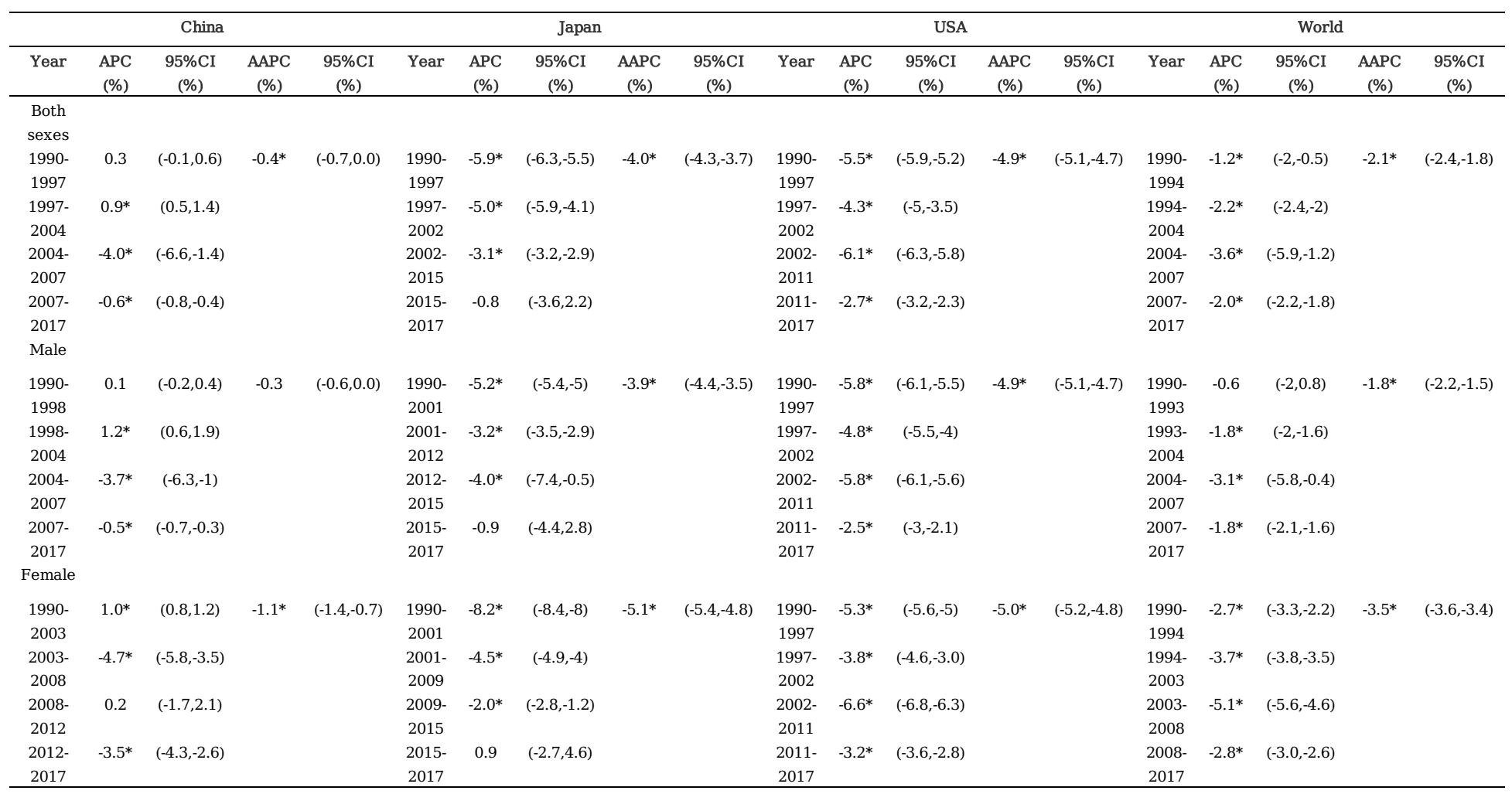

*: statistically significant ( $\mathrm{p}<0.05)$; APC: annual percent change; AAPC: average annual percent change.

Table 2. The temporal trend in mortality rate of CVD attributable to secondhand smoke in China, Japan, USA and the world from 1990 to 2017.

\begin{tabular}{|c|c|c|c|c|c|c|c|c|c|c|c|c|c|c|c|c|c|c|c|}
\hline \multicolumn{5}{|c|}{ China } & \multicolumn{5}{|c|}{ Japan } & \multicolumn{5}{|c|}{ USA } & \multicolumn{5}{|c|}{ World } \\
\hline Year & $\begin{array}{l}\text { APC } \\
(\%) \\
\end{array}$ & $\begin{array}{c}95 \% \mathrm{CI} \\
(\%) \\
\end{array}$ & $\begin{array}{c}\text { AAPC } \\
(\%)\end{array}$ & $\begin{array}{c}95 \% \mathrm{CI} \\
(\%) \\
\end{array}$ & Year & $\begin{array}{c}\text { APC } \\
(\%) \\
\end{array}$ & $\begin{array}{c}95 \% \mathrm{CI} \\
(\%) \\
\end{array}$ & $\begin{array}{c}\text { AAPC } \\
(\%)\end{array}$ & $\begin{array}{c}95 \% \mathrm{CI} \\
(\%) \\
\end{array}$ & Year & $\begin{array}{l}\text { APC } \\
(\%) \\
\end{array}$ & $\begin{array}{c}95 \% \mathrm{CI} \\
(\%) \\
\end{array}$ & $\begin{array}{c}\text { AAPC } \\
(\%)\end{array}$ & $\begin{array}{c}95 \% \mathrm{CI} \\
(\%) \\
\end{array}$ & Year & $\begin{array}{l}\text { APC } \\
(\%) \\
\end{array}$ & $\begin{array}{c}95 \% \mathrm{CI} \\
(\%)\end{array}$ & $\begin{array}{c}\text { AAPC } \\
(\%)\end{array}$ & $\begin{array}{c}95 \% \mathrm{CI} \\
(\%) \\
\end{array}$ \\
\hline $\begin{array}{c}\text { Both } \\
\text { sexes }\end{array}$ & & & & & & & & & & & & & & & & & & & \\
\hline $\begin{array}{l}1990- \\
1999\end{array}$ & $-2.0^{*}$ & $(-2.4,-1.6)$ & $-1.2^{*}$ & $(-1.7,-0.7)$ & $\begin{array}{l}1990- \\
2002\end{array}$ & $-5.3^{*}$ & $(-5.5,-5.2)$ & $-3.8^{*}$ & $(-4.1,-3.5)$ & $\begin{array}{c}1990- \\
2000\end{array}$ & $-4.2^{*}$ & $(-4.5,-4)$ & $-3.9 *$ & $(-4.2,-3.6)$ & $\begin{array}{l}1990- \\
1994\end{array}$ & $-1.1^{*}$ & $(-1.7,-0.5)$ & $-1.8^{*}$ & $(-2.0,-1.5)$ \\
\hline $\begin{array}{l}1999- \\
2004\end{array}$ & 0.4 & $(-0.9,1.7)$ & & & $\begin{array}{l}2002- \\
2011\end{array}$ & $-2.2^{*}$ & $(-2.6,-1.9)$ & & & $\begin{array}{l}2000- \\
2007\end{array}$ & $-6.6^{*}$ & $(-7.2,-6)$ & & & $\begin{array}{l}1994- \\
2004\end{array}$ & $-1.9^{*}$ & $(-2.1,-1.7)$ & & \\
\hline $\begin{array}{l}2004- \\
2007\end{array}$ & -2.7 & $(-6.6,1.3)$ & & & $\begin{array}{l}2011- \\
2015\end{array}$ & $-3.9^{*}$ & $(-5.4,-2.4)$ & & & $\begin{array}{l}2007- \\
2012\end{array}$ & $-3.6^{*}$ & $(-4.7,-2.5)$ & & & $\begin{array}{l}2004- \\
2007\end{array}$ & $-3.3^{*}$ & $(-5.1,-1.4)$ & & \\
\hline $\begin{array}{l}2007- \\
2017\end{array}$ & $-0.7^{*}$ & $(-1,-0.4)$ & & & $\begin{array}{l}2015- \\
2017\end{array}$ & -1.7 & $(-4.6,1.4)$ & & & $\begin{array}{l}2012- \\
2017\end{array}$ & 0.4 & $(-0.5,1.2)$ & & & $\begin{array}{l}2007- \\
2017\end{array}$ & $-1.4^{*}$ & $(-1.6,-1.3)$ & & \\
\hline Male & & & & & & & & & & & & & & & & & & & \\
\hline $\begin{array}{l}1990- \\
2000\end{array}$ & $-1.1^{*}$ & $(-1.5,-0.7)$ & -0.6 & $(-1.3,0.2)$ & $\begin{array}{l}1990- \\
2001\end{array}$ & $-3.7^{*}$ & $(-3.9,-3.4)$ & $-2.7 *$ & $(-3.1,-2.3)$ & $\begin{array}{l}1990- \\
1995\end{array}$ & $-4.0^{*}$ & $(-4.7,-3.3)$ & $-4.1 *$ & $(-4.4,-3.8)$ & $\begin{array}{l}1990- \\
1993\end{array}$ & -0.8 & $(-1.8,0.3)$ & $-1.5^{*}$ & $(-1.8,-1.3)$ \\
\hline $\begin{array}{l}2000- \\
2003\end{array}$ & 2.3 & $(-2.6,7.3)$ & & & $\begin{array}{l}2001- \\
2011\end{array}$ & $-1.3^{*}$ & $(-1.6,-1)$ & & & $\begin{array}{l}1995- \\
2009\end{array}$ & $-6.1^{*}$ & $(-6.2,-5.9)$ & & & $\begin{array}{l}1993- \\
2004\end{array}$ & $-1.7^{*}$ & $(-1.9,-1.6)$ & & \\
\hline $\begin{array}{l}2003- \\
2006\end{array}$ & -1.6 & $(-6.2,3.3)$ & & & $\begin{array}{l}2011- \\
2015\end{array}$ & $-4.1^{*}$ & $(-5.8,-2.4)$ & & & $\begin{array}{l}2009- \\
2014\end{array}$ & $-1.8^{*}$ & $(-2.8,-0.8)$ & & & $\begin{array}{l}2004- \\
2007\end{array}$ & $-2.3^{*}$ & $(-4.3,-0.3)$ & & \\
\hline $\begin{array}{l}2006- \\
2017\end{array}$ & $-0.5^{*}$ & $(-0.9,-0.2)$ & & & $\begin{array}{l}2015- \\
2017\end{array}$ & -1.6 & $(-5,1.9)$ & & & $\begin{array}{l}2014- \\
2017\end{array}$ & 1.5 & $(-0.1,3.1)$ & & & $\begin{array}{l}2007- \\
2017\end{array}$ & $-1.2^{*}$ & $(-1.4,-1.1)$ & & \\
\hline Female & & & & & & & & & & & & & & & & & & & \\
\hline $\begin{array}{l}1990- \\
1999\end{array}$ & $-2.4^{*}$ & $(-2.8,-2)$ & $-1.6^{*}$ & $(-2.1,-1.0)$ & $\begin{array}{l}1990- \\
1992\end{array}$ & $-5.3^{*}$ & $(-8.7,-1.7)$ & $-4.8 *$ & $(-5.1,-4.4)$ & $\begin{array}{l}1990- \\
2001\end{array}$ & $-3.1 *$ & $(-3.3,-2.9)$ & $-3.5^{*}$ & $(-3.8,-3.3)$ & $\begin{array}{l}1990- \\
1994\end{array}$ & $-1.0^{*}$ & $(-1.7,-0.4)$ & $-1.9^{*}$ & $(-2.1,-1.8)$ \\
\hline $\begin{array}{l}1999- \\
2004\end{array}$ & 0 & $(-1.5,1.5)$ & & & $\begin{array}{l}1992- \\
2002\end{array}$ & $-6.8^{*}$ & $(-7.2,-6.5)$ & & & $\begin{array}{l}2001- \\
2007\end{array}$ & $-7.1^{*}$ & $(-7.8,-6.5)$ & & & $\begin{array}{l}1994- \\
2004\end{array}$ & $-2.0^{*}$ & $(-2.2,-1.8)$ & & \\
\hline $\begin{array}{l}2004- \\
2007\end{array}$ & -3.7 & $(-8.1,1)$ & & & $\begin{array}{c}2002- \\
2015\end{array}$ & $-3.5^{*}$ & $(-3.7,-3.3)$ & & & $\begin{array}{l}2007- \\
2012\end{array}$ & $-3.6^{*}$ & $(-4.5,-2.7)$ & & & $\begin{array}{l}2004- \\
2008\end{array}$ & $-3.6^{*}$ & $(-4.6,-2.6)$ & & \\
\hline $\begin{array}{l}2007- \\
2017\end{array}$ & $-0.9 *$ & $(-1.3,-0.5)$ & & & $\begin{array}{l}2015- \\
2017\end{array}$ & -2 & $(-5.6,1.7)$ & & & $\begin{array}{l}2012- \\
2017\end{array}$ & 0 & $(-0.7,0.7)$ & & & $\begin{array}{l}2008- \\
2017\end{array}$ & $-1.5^{*}$ & $(-1.7,-1.3)$ & & \\
\hline
\end{tabular}

*: statistically significant ( $\mathrm{p}<0.05)$; APC: annual percent change; AAPC: average annual percent change. 
Table 3. The net drift value of the mortality rate of CVD attributable to Tobacco Exposure

\begin{tabular}{|c|c|c|c|c|c|c|c|c|c|}
\hline & & \multicolumn{2}{|c|}{ China } & \multicolumn{2}{|c|}{ Japan } & \multicolumn{2}{|c|}{ United States } & \multicolumn{2}{|c|}{ Global } \\
\hline & & $\begin{array}{c}\text { Net Drift } \\
\text { (\%/year) }\end{array}$ & $95 \% \mathrm{CI}$ & $\begin{array}{c}\text { Net Drift } \\
\text { (\%/year) }\end{array}$ & $95 \% \mathrm{CI}$ & $\begin{array}{l}\text { Net Drift } \\
\text { (\%/year) }\end{array}$ & $95 \% \mathrm{CI}$ & $\begin{array}{l}\text { Net Drift } \\
\text { (\%/year) }\end{array}$ & $95 \% \mathrm{CI}$ \\
\hline \multirow[t]{3}{*}{ Smoking } & both & -1.049 & $(-0.837,-1.26)$ & -2.983 & $(-2.855,-3.11)$ & -4.341 & $(-4.25,-4.433)$ & -2.126 & $(-2.085,-2.167)$ \\
\hline & $\begin{array}{l}\text { sexes } \\
\text { male }\end{array}$ & -0.847 & $(-0.624,-1.071)$ & -2.895 & $(-2.8,-2.989)$ & -4.359 & $(-4.256,-4.462)$ & -1.845 & $(-1.803,-1.888)$ \\
\hline & female & -2.507 & $(-1.817,-3.192)$ & -3.774 & $(-3.458,-4.089)$ & -4.379 & $(-4.259,-4.499)$ & -3.593 & $(-3.488,-3.698)$ \\
\hline \multirow[t]{3}{*}{$\begin{array}{l}\text { Secondhand } \\
\text { smoke }\end{array}$} & $\begin{array}{l}\text { both } \\
\text { sexes }\end{array}$ & -2.247 & $(-2.028,-2.466)$ & -2.632 & $(-2.389,-2.875)$ & -3.871 & $(-3.742,-4.001)$ & -1.969 & $(-1.918,-2.019)$ \\
\hline & male & -1.030 & $(-0.792,-1.267)$ & -1.898 & $(-1.59,-2.206)$ & -4.026 & $(-3.871,-4.18)$ & -1.574 & $(-1.517,-1.631)$ \\
\hline & female & -3.135 & $(-2.906,-3.364)$ & -3.698 & $(-3.298,-4.097)$ & -3.602 & $(-3.365,-3.838)$ & -2.340 & $(-2.271,-2.408)$ \\
\hline
\end{tabular}

\section{Additional Files}

Additional file 1:

Table S1. The prevalence of smoking in four regions from 2000 to 2015

Table S2. The temporal trend in mortality rate of stroke attributable to smoking in China, Japan, USA and the world from 1990 to 2017.

Table S3. The temporal trend in mortality rate of stroke attributable to secondhand smoke in China, Japan, USA and the world from 1990 to 2017.

Table S4. The net drift value of the mortality rate of stroke attributable to Tobacco Exposure

Table S5. The temporal trend in mortality rate of IHD attributable to smoking in China, Japan, USA and the world from 1990 to 2017.

Table S6. The temporal trend in mortality rate of IHD attributable to secondhand smoke in China, Japan, USA and the world from 1990 to 2017.

Table S7. The net drift value of the mortality rate of IHD attributable to Tobacco Exposure

\section{Additional file 2:}

Figure S1. The ASMR of stroke attributable to smoking in China, Japan, USA and the world from 1990 to 2017

Figure S2. The ASMR of stroke attributable to secondhand smoke in China, Japan, USA and the world from 1990 to 2017

Figure S3. The APC results of stroke attributable to smoking in China, Japan, USA and the world from 1990 to 2017

Figure S4. The local drift with net drift values of the mortality rate of stroke attributable to tobacco exposure in China, Japan, USA and the world from 1990 to 2017

Figure S5. The APC results of stroke attributable to secondhand smoke in China, Japan, USA and the world from 1990 to 2017

Figure S6. The ASMR of IHD attributable to smoking in China, Japan, USA and the world from 1990 to 2017

Figure S7. The ASMR of IHD attributable to secondhand smoke in China, Japan, USA and the world from 1990 to 2017

Figure S8. The APC results of IHD attributable to smoking in China, Japan, USA and the world from 1990 to 2017

Figure S9. The local drift with net drift values of the mortality rate of IHD attributable to tobacco exposure in China, Japan, USA and the world from 1990 to 2017

Figure S10. The APC results of IHD attributable to secondhand smoke in China, Japan, USA and the world from 1990 to 2017

\section{Figures}



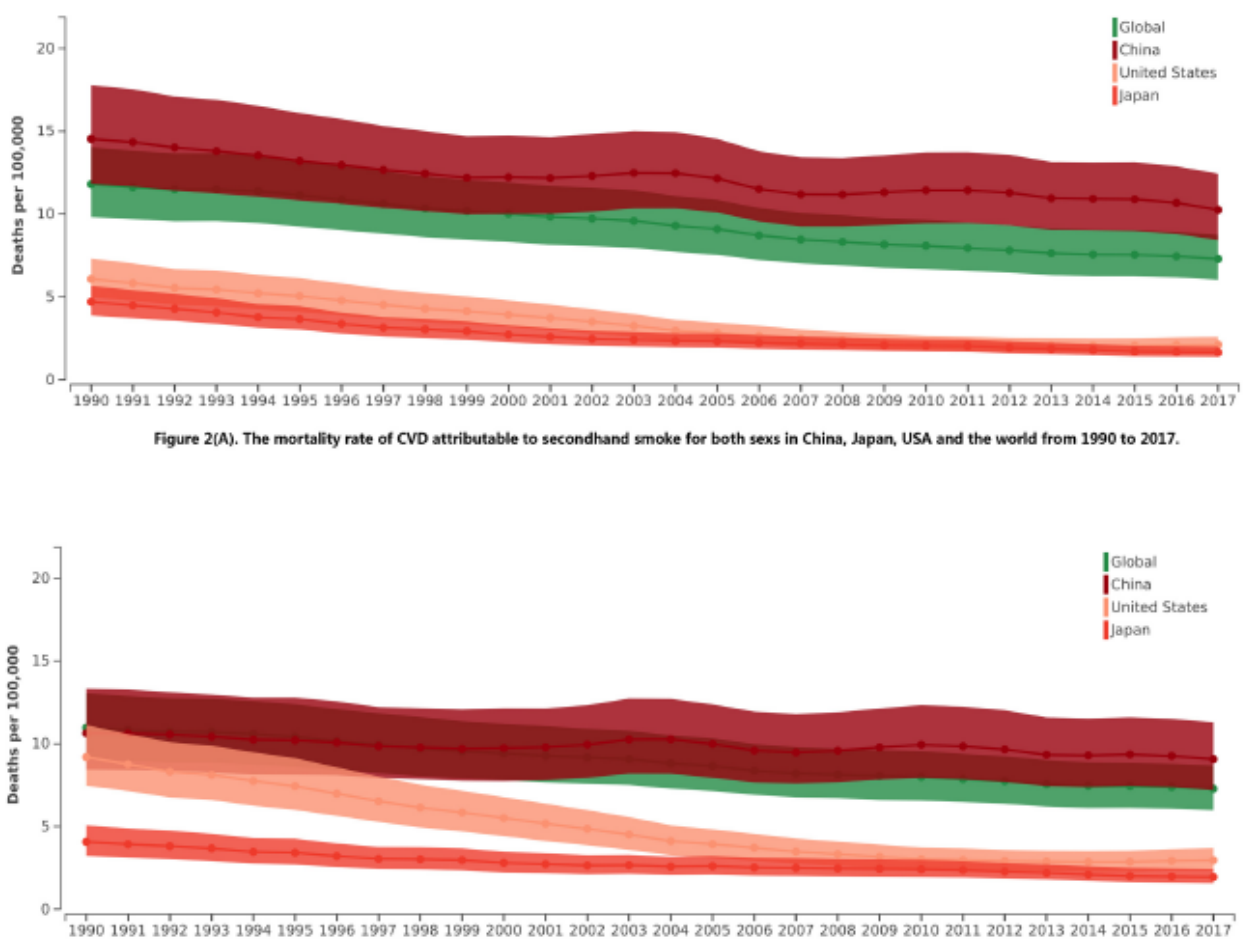

Figure 2(B). The mortality rate of CVD attributable to secondhand smoke for male in China, Japan, USA and the world from 1990 to 2017.

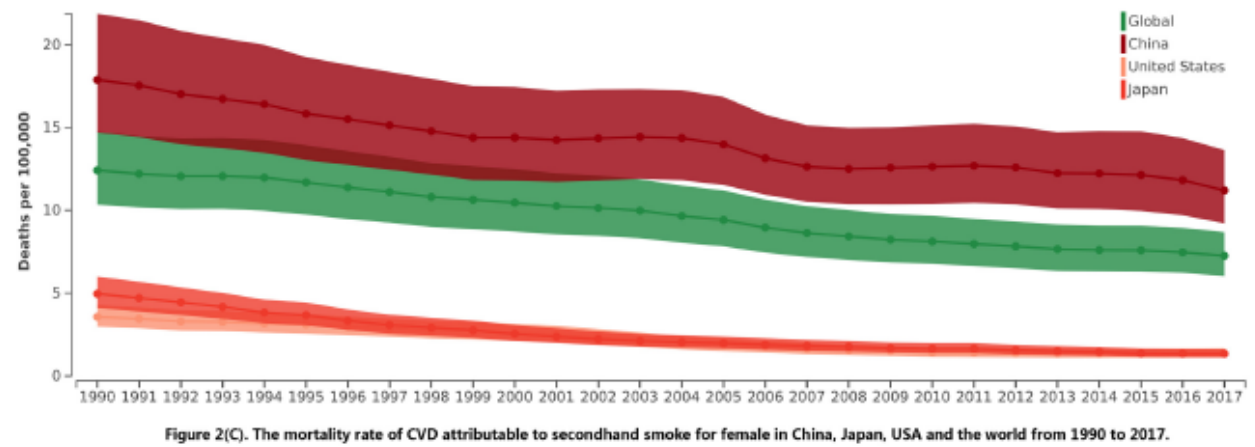

Figure 1

The ASMR of CVD attributable to smoking in China, Japan, USA and the world from 1990 to 2017 

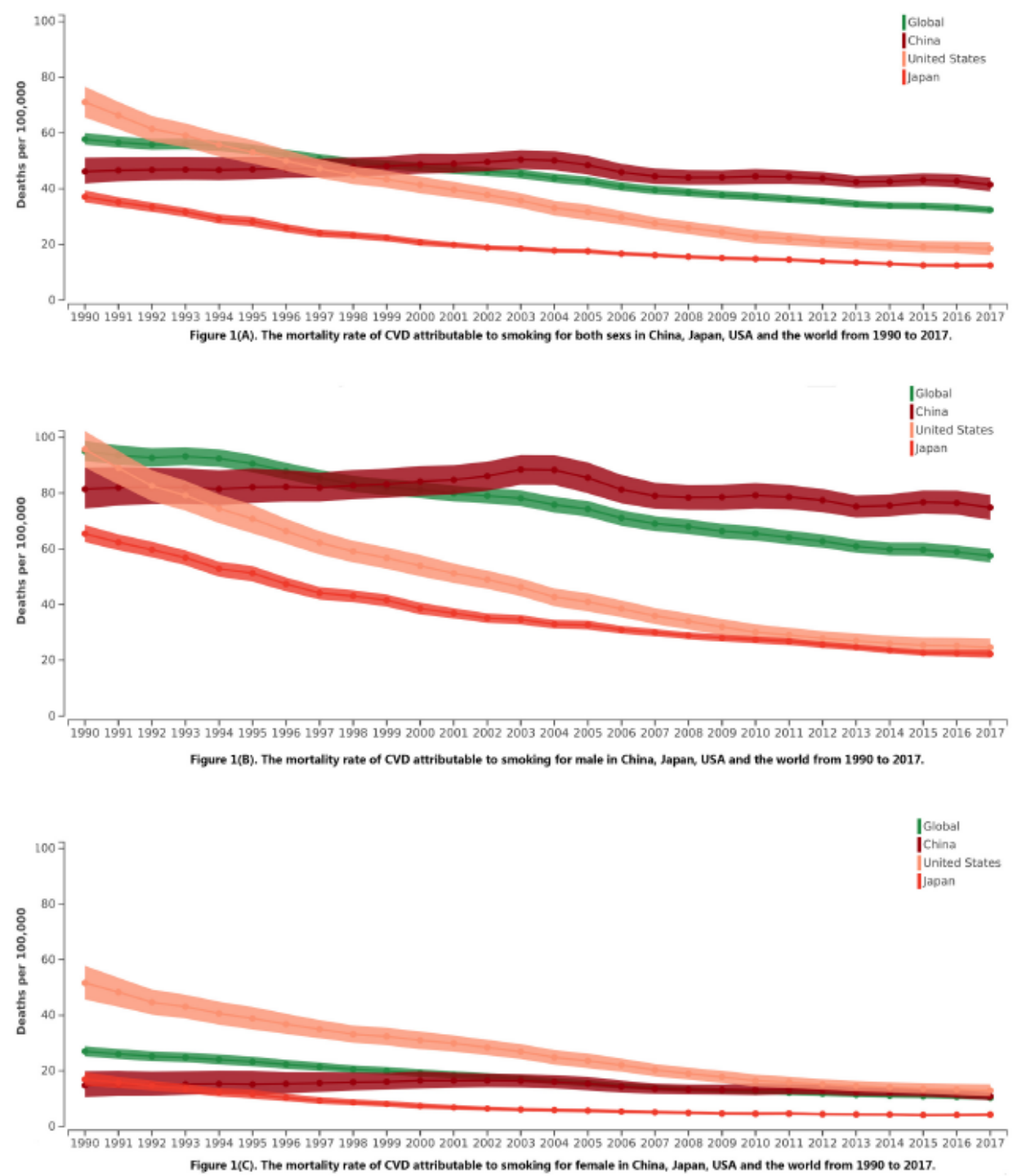

Figure 2

The ASMR of CVD attributable to Secondhand smoke in China, Japan, USA and the world from 1990 to 2017 

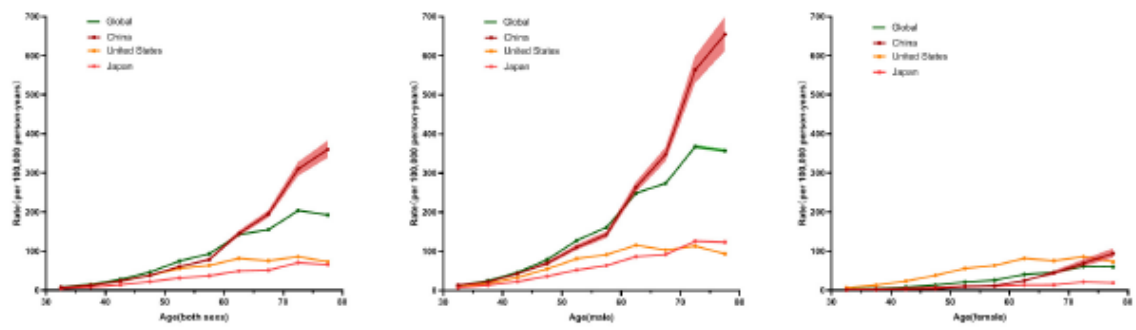

Figure 3(A). the longitudinal age curves of the mortality rate of CVD attributable to smoking in global, China, United States and Japan.
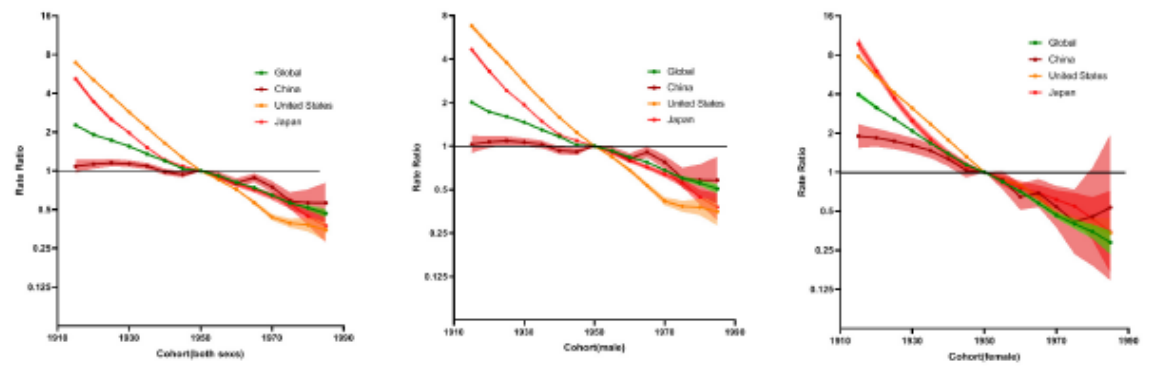

Figure 3(B). The estimated cohort RRs of of the mortality rate of CVD attributable to smoking in global, China, United States and Japan.
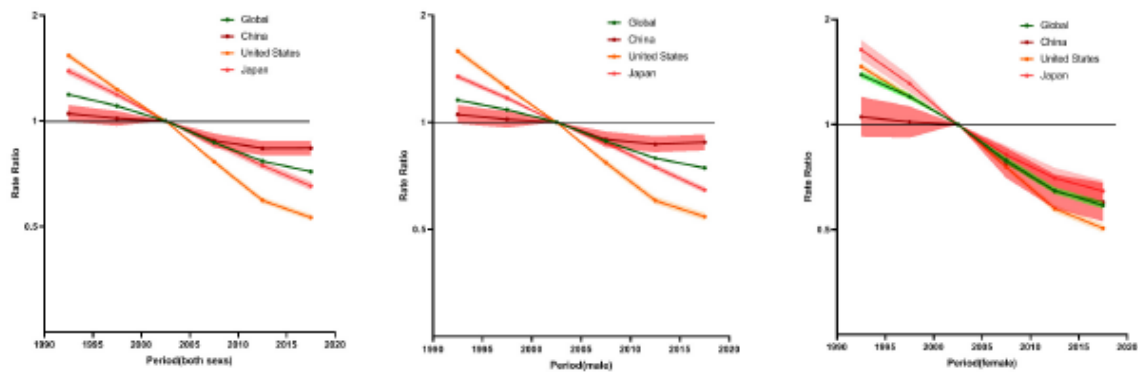

Figure $3(\mathrm{C})$. The estimated period RRs of of the mortality rate of CVD attributable to smoking in global, China, United States and Japan.

Figure 3

The APC results of CVD attributable to smoking in China, Japan, USA and the world from 1990 to 2017. 

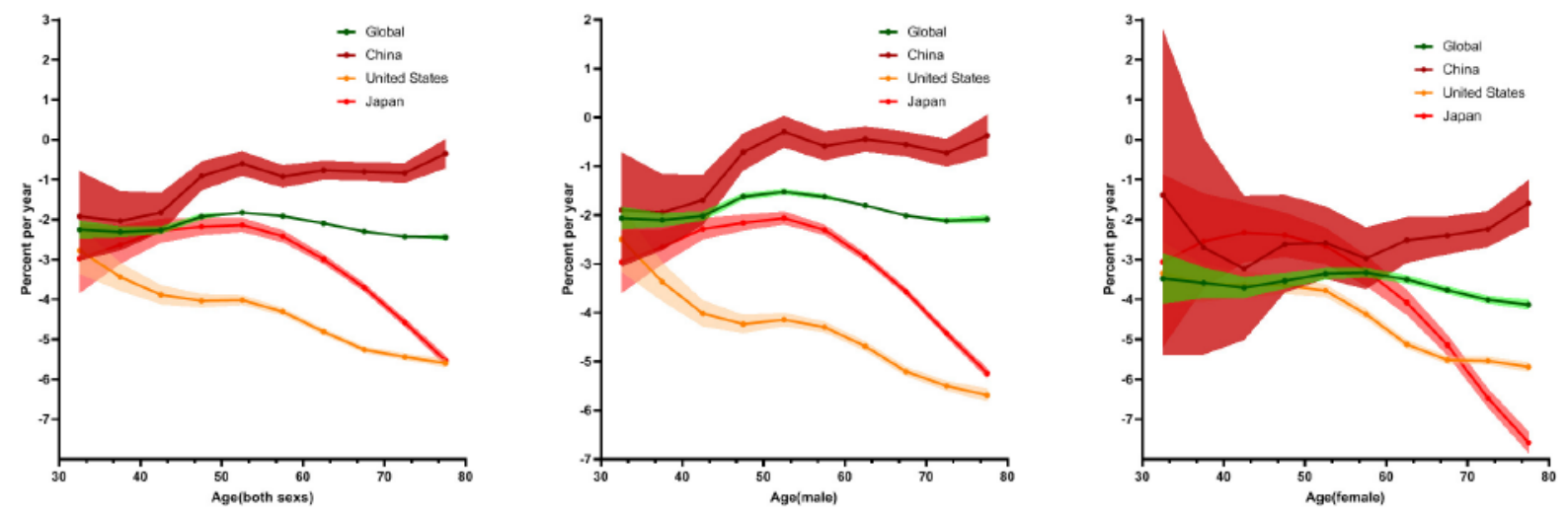

Figure 4(A). The local drift with net drift values of the mortality rate of CVD attributable to smoking in global, China, United States and Japan.
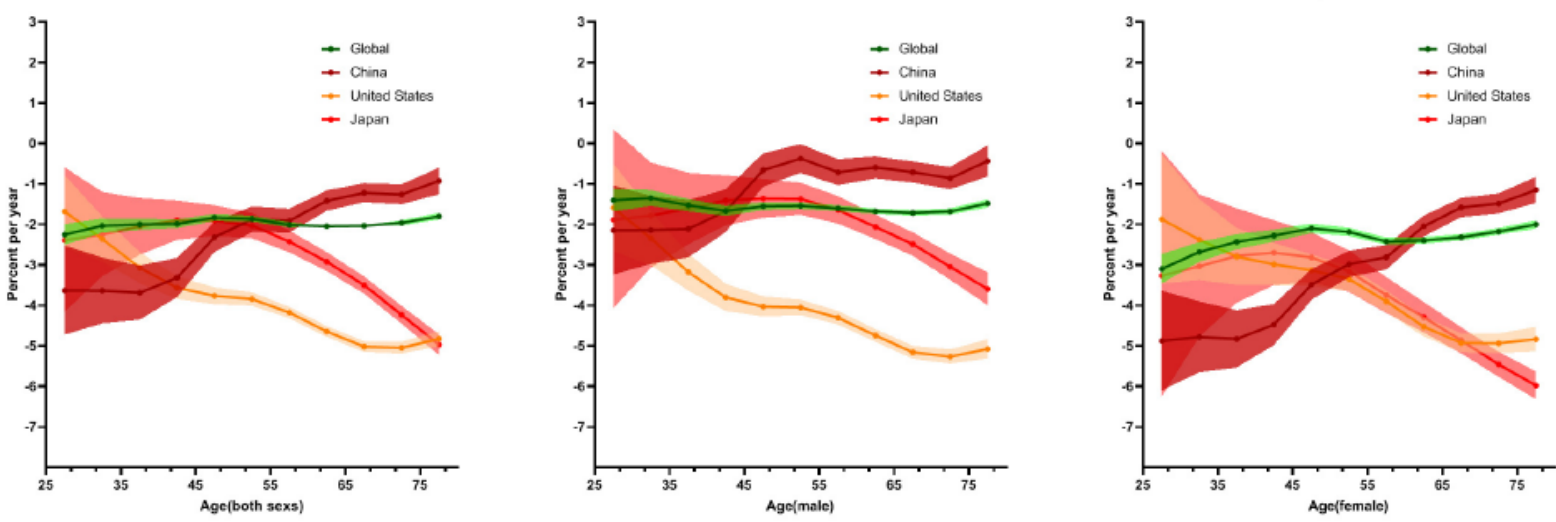

Figure 4(B). The local drift with net drift values of the mortality rate of CVD attributable to secondhand smoke in global, China, United States and Japan.

\section{Figure 4}

The local drift with net drift values of the mortality rate of CVD attributable to tobacco exposure in China, Japan, USA and the world from 1990 to 2017 

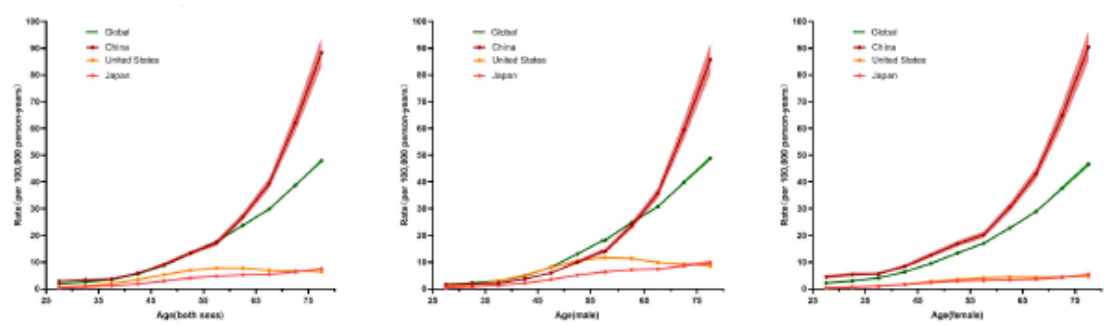

Figure 5(A). the longitudinal age curves of the mortality rate of CVD attributable to secondhand smoke in global, China, United States and Japan.
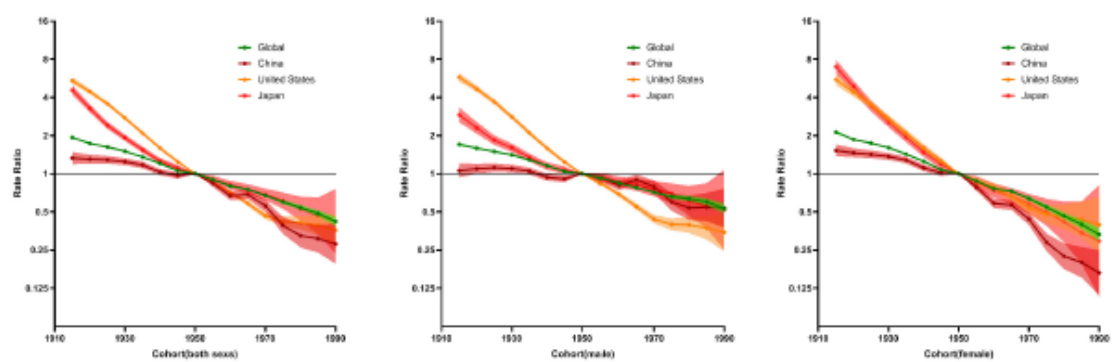

Figure 5(B). The estimated cohort RRs of of the mortality rate of CVD attributable to secondhand smoke in global, China, United States and Japan.
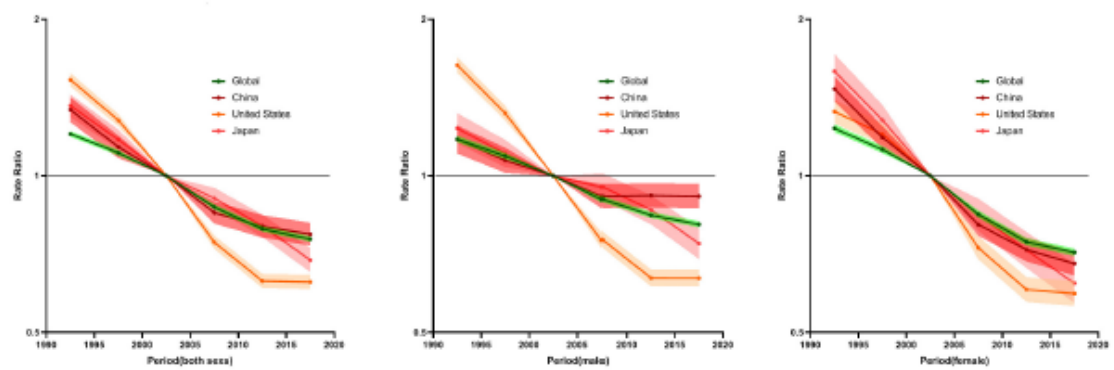

Figure $5(C)$. The estimated period RRs of of the mortality rate of CVD attributable to secondhand smoke in global, China, United States and Japan.

Figure 5

The APC results of CVD attributable to secondhand smoke in China, Japan, USA and the world from 1990 to 2017.

\section{Supplementary Files}

This is a list of supplementary files associated with this preprint. Click to download.

- Additionalfile1.pdf

- Additionalfile2.pdf 